\title{
Thin Films for Magneto-Optical Applications
}

\author{
Manabu GOMI \\ Department of Electrical and Electronic Engineering, Tokyo Institute of Technology, 2-12-1, Ookayama, Meguro-ku, Tokyo 152, Japan
}

\section{磁気光学用薄膜}

五味 学

東京工業大学電気電子工学科, 152 東京都目黒区大岡山 2-12-1

\begin{abstract}
本論文では, 最初に磁気光学効果の現象論, 微視的理論を簡単に概観し, 磁気光学的応用に有用な磁性薄膜の作 製及び磁気光学的性質に関する最近の研究を紹介する，そこでは，薄膜への $\mathrm{Bi}^{3+}$ のようなイオンの多量置換が 磁気光学効果を増大させるのに重要であることが強調され，この多量置換はスパッタ法のような熱的非平衡下で 膜を作製することにより達成されることが述べられる。本論文で取り扱われた磁気光学用薄膜材料は, Bi 及び 軽希土類置換ガーネットフェライト, $\mathrm{Co}^{2+}$ を含むスピネル及び六方晶フェライト, 磁性半導体, 強磁性金属で ある。
\end{abstract}

[Received April 24, 1991]

\begin{abstract}
Some recent works on preparation and magneto-optical (MO) properties of magnetic thin films useful for MO applications are reviewed after briefly outlining phenomenological and microscopic theory of MO effect. It is emphasized that high substitution of specific ions such as $\mathrm{Bi}^{3+}$ to the film is important to strongly enhance the MO effect. This is achieved by making the film under thermally non-equilibrium state such as sputtering method. The classes of the MO thin films discussed are Bi- and light rare earth ions-substituted garnet ferrites, spinel and hexagonal ferrites containing $\mathrm{Co}^{2+}$, magnetic semiconductor and ferromagnetic metals.
\end{abstract}

Key-words : Magneto-optical thin film, Magneto-optical effect, Magneto-optical enhancement, Bi-substituted iron garnet, Spinel ferrite, Hexagonal ferrite, Sputtering method

\section{Introduction}

$\mathbf{F}$ unctional optical devices utilizing electro-optical or magneto-optical (MO) effects will occupy a significant part in electronics in the future. In a very wide meaning, the MO effect denotes phenomena that electromagnetic wave propagating through material or reflected at the surface of the material is changed not only in polarized state but also in magnitude by applied external megnetic field $\boldsymbol{H}$ or magnetization $\boldsymbol{M}$ of the material. Among them the strongest MO effects proportional to first order of $\boldsymbol{H}$ or $\boldsymbol{M}$, called Faraday effect or Kerr effect, have attracted much attention for practical applications. These effects were also of academic interest because we could reveal the electronic structure of the magnetic material by MO spectroscopy.

The MO effect in the visible region has been first applied to the MO memory device in $1962,{ }^{1}$ in which the thin film of a MO material was used as a storage medium and the information written in it was retrieved by the MO effect. This idea has been put to practical use at present as a MO disc for high density magnetic recording. On the other hand, the development of the optical communication system requires some optically functional components using MO effect such as non-reciprocal circuit and isolator, though those have been obtained in microwave region. Those MO components are desired to be miniaturized and further integrated into optical circuits in the fufure.

Thus, it is essential to make thin films of the MO materials for applications. This is also required from a fact that, in general, the MO materials exhibit strong optical absorption as well as large MO effect in the visible region, which is described later.

The purpose of the present paper is to review some recent works on the magnetic thin films useful for MO applications. We first outline phenomenological theory and microscopic origin of the MO effect briefly. Secondly we describe preparation and MO properties of the MO thin films exhibiting pronounced MO effect and/or useful for practical applications.

\section{Phenomenology of MO effect}

When, in optically isotropic materials, the applied external magnetic field $\boldsymbol{H}$ or the magnetization $\boldsymbol{M}$ of the material lies along $z$ axis, we can express the complex dielectric tensor of the material as follows: ${ }^{2)}$

$$
\mathcal{E}=\left(\begin{array}{ccc}
\varepsilon_{x x} & \varepsilon_{x y} & 0 \\
-\varepsilon_{x y} & \varepsilon_{x x} & 0 \\
0 & 0 & \varepsilon_{x x}
\end{array}\right)
$$

The $\varepsilon_{x y}$ is the term induced by the applied external magnetic field and/or the magnetization of the material, an odd function of the $z$ component of $\boldsymbol{H}$ (or $\boldsymbol{M})$ whereas the $\varepsilon_{x x}$ is an even function of them. Here, 


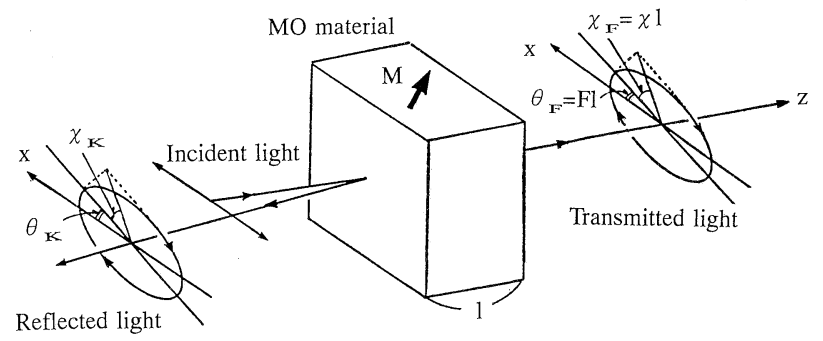

Fig. 1. Faraday effect and Kerr effect.

$\varepsilon_{x x}$ and $\varepsilon_{x y}$ have a real and an imaginary part:

$$
\begin{aligned}
& \varepsilon_{x x}=\varepsilon_{x x}{ }^{\prime}+i \varepsilon_{x x}{ }^{\prime \prime} \\
& \varepsilon_{x y}=\varepsilon_{x y}{ }^{\prime}+i \varepsilon_{x y}{ }^{\prime \prime}
\end{aligned}
$$

$\varepsilon_{x x}{ }^{\prime \prime}$ is responsible for optical absorption of the material. The light propagating along the $z$ axis of the material, then, is represented as a linear combination of two unique modes, i.e. two circularly polarized lights which has electric vector rotating in the opposite direction each other at a given point in space. The complex refractive indices $n^{ \pm}$of the material for the respective circularly polarized lights are given as follows;

$$
\left(n^{ \pm}\right)^{2}=\varepsilon_{x x} \pm i \varepsilon_{x y}
$$

Since the propagation velocity is different between two circularly polarized lights, therefore, the light transmitted through the material is elliptically polarized with the major axis of the ellipse rotated by an angle relative to the incident light (Fig. 1). This is called Faraday effect. Then, we obtain the following Faraday rotation $F$ and Faraday ellipticity $\chi$ which are defined as the rotation angle and ellipticity per unit length of the MO material, respectively,

$$
\begin{aligned}
F+i \chi & =(\pi / \lambda)\left(n^{+}-n^{-}\right) \\
& =(\pi / \lambda) i \varepsilon_{x y} /\left(\varepsilon_{x x}\right)^{1 / 2}
\end{aligned}
$$

where $\varepsilon_{x x} \gg \varepsilon_{x y}$, and $\lambda$ is the wavelength of the light in vacuum.

When the linearly polarized light is normally incident on the MO material, the light reflected on the surface of the MO material also undergoes similar MO effect because the material has Fresnel's coefficients of $r^{ \pm}=\left(n^{ \pm}-1\right) /\left(n^{ \pm}+1\right)$ different for the above two unique modes. This is called polar Kerr effect, the situation of which is shown in Fig. 1. The polar Kerr rotation angle $\theta_{\mathrm{K}}$ and the Kerr ellipticity $\chi_{\mathrm{K}}$ of the reflected light is approximately calculated, when $\theta_{\mathrm{K}}, \chi_{\mathrm{K}} \ll 1$, as follows:

$$
\theta_{\mathrm{K}}+i \chi_{\mathrm{K}}=-\varepsilon_{x y} /\left\{\left(\varepsilon_{x x}-1\right)\left(\varepsilon_{x x}\right)^{1 / 2}\right\} .
$$

Thus the Eqs. (4), (5) tell us that, when $\varepsilon_{x x}{ }^{\prime \prime} \ll \varepsilon_{x x}{ }^{\prime}$, Faraday and Kerr rotation are proportinal to the $\varepsilon_{x y}{ }^{\prime \prime}$ and $\varepsilon_{x y}{ }^{\prime}$ respectively.

\section{Microscopic origin of MO effect}

The above dielectric tensor is related to general atomic parameters characteristic of energy levels such as level separation, linewidth, transition probability, etc. If a light propagates a solid, an ion in the solid will exhibit an electric dipole moment due to an electric field $\boldsymbol{E}$ of the light. The ratio of the electric di- pole moment to $\boldsymbol{E}$ is called the polarizability. If a transition from a ground energy level labeled $a$ to a higher energy level labeled $b$ by a light are supposed for an electron attached to an ion, the components of the polarizability are given by Condon and Shortley $\mathrm{as}^{3)}$

$$
\begin{aligned}
\alpha_{i j}= & \sum_{a} \beta_{a} \frac{1}{\hbar} \sum_{b}\left[\frac{P_{a b}^{i} P_{b a}^{j}}{\omega_{a b}+\omega-i \Gamma_{a b}}\right. \\
& \left.+\frac{P_{a b} P_{b a}^{i}}{\omega_{a b}-\omega+i \Gamma_{a b}}\right]
\end{aligned}
$$

where $i, j=x, y, z$ and $\omega_{a b}$ and $\Gamma_{a b}$ are the energy gap and the linewidth of the $a b$ transition, respectively. $\beta_{a}$ denotes the probability that an electron is in the level $a$. The $P_{a b}{ }^{i}$ gives the probability that an electron will make a transition from $a$ to $b$. Then the off diagonal term $\varepsilon_{x y}$ is expressed by

$$
\begin{aligned}
\varepsilon_{x y}=4 \pi N \alpha_{x y}= & \frac{2 \pi N e^{2}}{m} \sum_{a, b} \frac{\beta_{a}\left(f_{a b}{ }^{+}-f_{a b}{ }^{-}\right)}{\omega_{a b}} \\
& \cdot \frac{i \omega+\Gamma_{a b}}{\omega_{a b}{ }^{2}-\omega^{2}+\Gamma_{a b}{ }^{2}+i 2 \omega \Gamma_{a b}}
\end{aligned}
$$

here $N$ is the numbers of ions per $\mathrm{cm}^{3}$ and $m$ and $e$ are the mass and charge of the electron. We have used the definition

$$
f_{a b}^{ \pm}=\frac{m \omega_{a b}}{\hbar e^{2}}\left|P_{a b}{ }^{x} \pm i P_{a b}\right|^{2}
$$

which is called the oscillator strength for right circular polarized light $(+)$ or left circular polarized light $(-)$.

For insulator in which electrons are localized, we can concretely derive dispersion relations of $\varepsilon_{x y}{ }^{\prime \prime}$ responsible for Faraday rotation, in case of two electronic transitions shown in Fig. 2(a) and Fig. 3(a). Figure $2(\mathrm{a})$ shows the case that $a b$ transition is allowed only for the right circular polarized light. At low temperature where all electrons are condensed into the lowest ground level, the Eq. (7) gives

$$
\varepsilon_{x y}{ }^{\prime \prime}=-\frac{2 \pi N e^{2}}{m \omega_{0}} \cdot f \cdot \frac{\omega\left(\omega^{2}-\omega_{0}^{2}+\Gamma^{2}\right)}{\left(\omega_{0}^{2}-\omega^{2}+\Gamma^{2}\right)^{2}+4 \Gamma^{2} \omega^{2}} .
$$

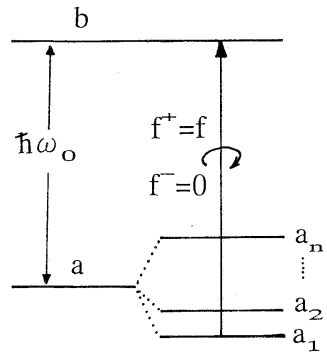

(a)

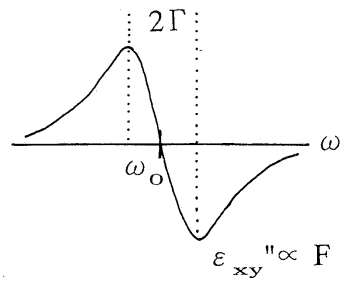

(b)
Fig. 2. (a) Energy levels for single transitions and (b) single-transition line shape. 


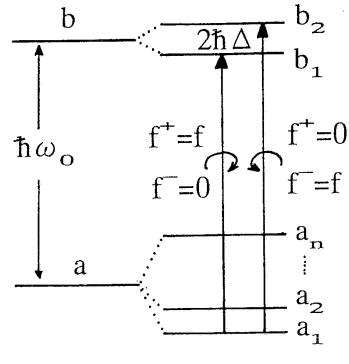

(a)

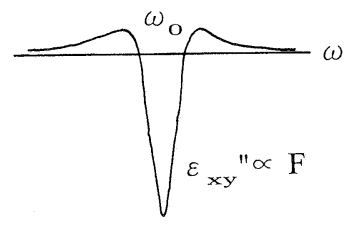

(b)
Fig. 3. (a) Energy levels for double transitions and (b) doubletransition line shape.

Faraday rotation has, therefore, a dispersive line shape, proportional to the oscillator strength (Fig. 2 (b)). This type of rotation depends on temperature because the electrons also occupy the upper ground level with rising temperature, therefore called a "paramagnetic rotation". In Fig. 3(a), the upper state are split by $2 \hbar \Delta$ by spin-orbit coupling. The $a_{1}$ $b_{2}$ transition absorbs only left circular polarized light, and the $a_{1}-b_{1}$ transition absorbs only right circular polarized light. The $\varepsilon_{x y}{ }^{\prime \prime}$ is calculated as the superposition of two closely spaced curves expressed by the Eq. (7) when $\Delta \ll \Gamma \ll \omega$ :

$$
\begin{aligned}
\varepsilon_{x y}{ }^{\prime \prime}= & -\frac{2 \pi N e^{2}}{m \omega_{0}} \cdot f \Delta \cdot \\
& \frac{\left(\omega-\omega_{0}\right)^{2}-\Gamma^{2}}{\left[\left(\omega-\omega_{0}\right)^{2}-\Gamma^{2}\right]^{2}+4 \Gamma^{2}\left(\omega-\omega_{0}\right)^{2}}
\end{aligned}
$$

Consequently we can expect a large Faraday rotation in the materials with large $\Delta$ even when the oscillator strength $f$, i.e. optical absorption is weak. This line shape is dissipative, called "diamagnetic rotation". Thus, the materials exhibiting this type of electronic transition have high MO figure of merit defined later, much useful for MO applications.

In the materials with itinerant electrons such as metal, the above separated orbital approach breaks down. We must treat intra- and inter-band transition for discussing the MO effect. The MO effect comes from the difference of the state density between for up-spin electron and for down-spin electron, induced by spin-orbital interaction. ${ }^{4)}$ This is analogous to that for the localized electron described above. The reader should refer to the literature (Ref. 4) for details.

\section{Electronic transition and MO enhancement in MO materials}

The MO spectra of the practical materials show complicate structure in general since a number of electronic transitions overlap one another. As an example, Fig. 4 shows (a) diagonal elements and (b) off-diagonal elements of yttrium iron garnet $\mathrm{Y}_{3} \mathrm{Fe}_{5} \mathrm{O}_{12}$ (YIG) which is a typical ferrimagnetic oxide trans-

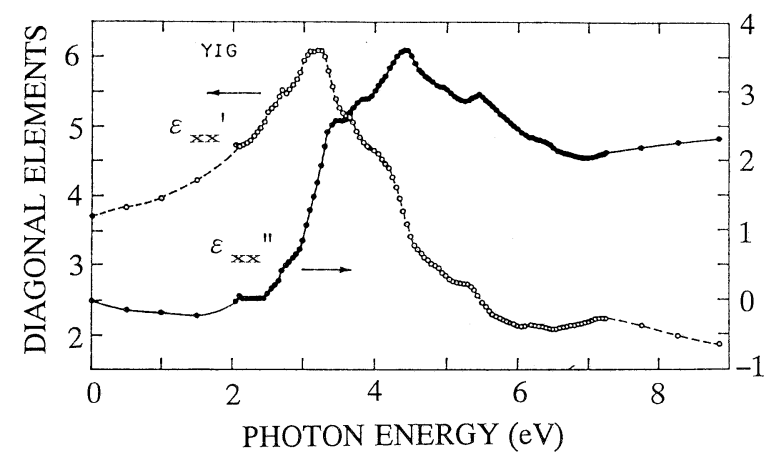

(a)

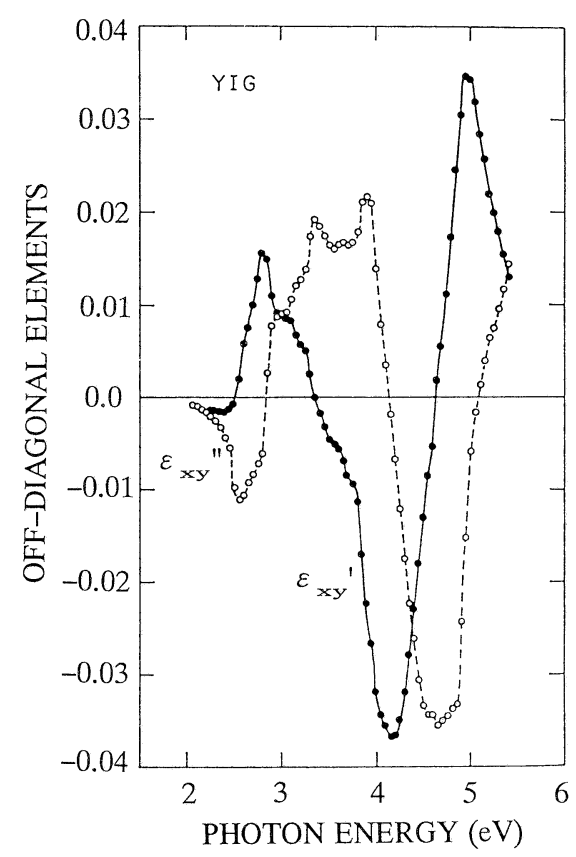

(b)

Fig. 4. (a) Complex diagonal elements $\varepsilon_{x x}=\varepsilon_{x x}{ }^{\prime}+i \varepsilon_{x x}{ }^{\prime \prime}$ and (b) offdiagonal elements $\varepsilon_{x y}=\varepsilon_{x y}{ }^{\prime}+i \varepsilon_{x y}$ " for yttrium iron garnet (from Ref. 5)).

parent in the visible and IR region. ${ }^{5)}$ It is known that the increases of $\varepsilon_{x x}{ }^{\prime \prime}$ (proportional to the optical absorption) and $\varepsilon_{x y}$ "at around $3 \mathrm{eV}$ are ascribed to charge transfer transition in which an electron of $\mathrm{Fe}^{3+}$ ion transfers to $\mathrm{O}^{2-}$. The charge transfer transition induces a strong MO effect and optical absorption because of allowed electric dipole transition with strong oscillator strength. The MO and optical absorption spectra caused by this transition extend to the visible region. However, in general, the optical absorption coefficient decreases much more than the MO effect at the wavelength away from the center of the electronic transition, which results in high MO figure of merit defined later. Small structure seen in $\varepsilon_{x x}{ }^{\prime \prime}$ at $2 \mathrm{eV}$ is due to crystal field transition which is an electronic transition between crystal field levels of $\mathrm{Fe}^{3+}$ split by the electric field of the anions. Since this transition is partially allowed, having a weak oscillator strength, it gives a small contribution on the 
MO effect. As other electronic transitions, orbital promotion transition is known, in which an electron bound in an orbital transfers to other orbital with larger quantum number. This transition shows extremely strong optical absorption and MO effect at higher photon energy than the charge transfer transition. ${ }^{3)}$

Some specific ions such as $\mathrm{Bi}^{3+}, \mathrm{Pr}^{3+}$ and $\mathrm{Co}^{2+}$ substituted to ferrites is known to enhance the MO effect just by the above electronic transitions. The typical examples are shown below.

The incorporation of $\mathrm{Bi}^{3+}$ ions in the garnet crystal $\mathrm{R}_{3} \mathrm{Fe}_{5} \mathrm{O}_{12} \quad(\mathrm{R}=$ rare earth ions or $\mathrm{Y})$ induces prominently strong MO effect as shown in Fig. 5.6) This enhancement is ascribed to the increase of the spin-orbit splitting in the excited state of $\mathrm{Fe}^{3+}-\mathrm{O}^{2-}$ charge transfer transition by orbital-mixing between $2 \mathrm{p}$ level of $\mathrm{O}^{2-}$ and $6 \mathrm{p}$ level of $\mathrm{Bi}^{3+}$ with large spin-orbit coupling constant. Though this transition is located in the UV, we can attain strong MO effect without increasing optical absorption in the visible region as described above.

The substitution of $\mathrm{Pr}^{3+}$ to the rare earth iron garnet induces pronounced MO effect by the $4 \mathrm{f}-5 \mathrm{~d}$ orbital promotion transition located in the UV as well. ${ }^{7)}$ The giant $\mathrm{MO}$ effect of EuO observed at low temperature ${ }^{3)}$ has been explained in terms of this transition in $\mathrm{Eu}^{2+}$.

It is found in many ferrites ${ }^{8)-10)}$ that the tetrahedrally coordinated $\mathrm{Co}^{2+}$ in ferrites induces large MO effect in the visible and IR originating from the crystal field transition as well. This is because the crystal field transition is partially allowed in the tetrahedron without center symmetry, having fairly strong oscillator strength.

To put the MO thin films to practical use, the films must have not only large MO effect but also low optical absorption coefficient $\alpha$ (in case of utilizing Faraday effect) or high optical reflection coefficient

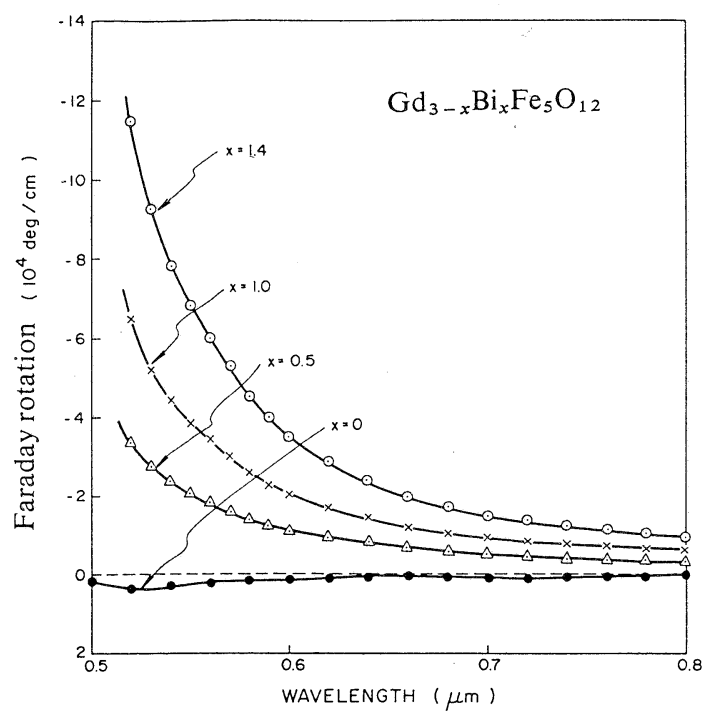

Fig. 5. Faraday spectra of Bi-substituted GdIG (from Ref. 6)).

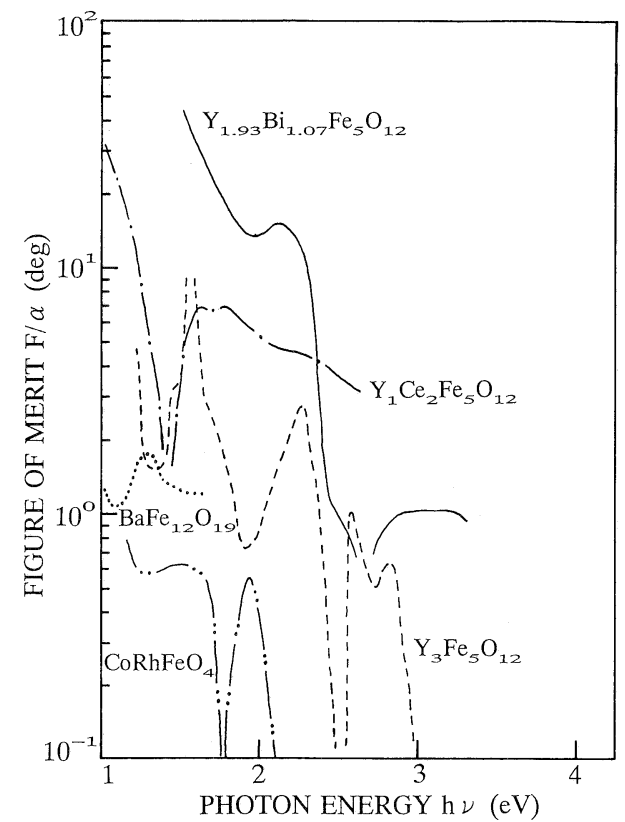

Fig. 6. Magneto-optical figure of merit for various ferrites.

$R$ (in case of utilizing Kerr effect). These are represented as significant parameters $F / \alpha$ or $R^{1 / 2} \theta_{\mathrm{K}}$, respectively, which are called MO figure of merit. ${ }^{2)}$ The materials containing the above specific ions show high MO figure of merit as shown in Fig. 6.11) Therefore many works on new MO materials have been devoted to the development of the films containig these enhancement ions.

\section{MO thin films and its characteristics}

In this section we describe growth techniques and characteristics of thin films exhibiting pronounced MO effect, i.e. containing a large amount of specific enhancement ions, or of new films useful for practical applications, developed until recently.

\subsection{Oxide thin films}

\subsubsection{Bi-substituted iron garnet films}

Since Bi-substituted iron garnet films have high MO figure of merit in the visible to the IR region as shown in Fig. 6, suitable for all MO applications, many works have been done on it. Bi-substituted iron garnet films have been prepared by Liquid Phase Epitaxial (LPE) method which was first used to get the garnet single crystal films with high magnetic quality for magnetic bubble memory. ${ }^{12)}$ The films are grown by dipping into a supersaturated solution consisting of the required garnet constituent oxides in a flux under truly isothermal conditions. ${ }^{13)}$ The most commonly employed flux system for LPE is $\mathrm{PbO}-\mathrm{B}_{2} \mathrm{O}_{3}$. This method is characterized by high reproducibility and much lower defect densities than other growing methods, suitable for producing defect-free single crystal films. However, the LPE films grown from a $\mathrm{PbO}$-based flux will incorporate a small amount of $\mathrm{Pb}^{2+}$ where it has to be charge compensated, often with $\mathrm{Pt}^{4+}$ from crucible. ${ }^{14)}$ In addi- 
tion, it is difficult to attain high Bi-substitution in the LPE films because ionic radius of $\mathrm{Bi}^{3+}$ is too large for the garnet lattice, which facilitates segregation of $\mathrm{Bi}$ ion from the garnet cryatal under the thermal equilibrium. These will be disadvantageous to MO applications.

Film growth of the garnet by sputtering method have been developed by several researchers. ${ }^{15)-17)}$ We can fabricate the sigle crystalline or polycrystalline film of the Bi-substituted iron garnet on substrates by crystallizing in situ during sputtering or by postannealing after depositing amorphous film. The sputtered garnet films have few contaminations with impurities but have generally many crystal imperfections produced by the bombardment of the growing film surface by the energetic negative ions and/or $\gamma$-electrons discharged from the target. However, Krumme et al. ${ }^{18)}$ have recently reported that the high structural quality is achieved by rf magnetron sputtering if the growing film is protected against energetic particle bombardment (the use of magnetron mode), and the targets without significant amounts of free $\mathrm{Bi}_{2} \mathrm{O}_{3}$ phase and the substrate with surface properly treated for epitaxy are used. The high-quality garnet films epitaxially sputtered under the above condition have lower optical absorption coefficient than the LPE films as shown in Fig. 7.18) As further evidence for the high structural quality it is shown that magnetic wall coercivity is as low as about 2 Oe at room temperature, comparable with that of the LPE film.

The sputtering method is also characterized by the growth conditions far from the thermodynamic equilibrium. This facilitates the film growth at low temperature, and also enables the synthesis of new materials with non-equilibrium compositions.

The synthesis of new iron garnet $\mathrm{Bi}_{3} \mathrm{Fe}_{5} \mathrm{O}_{12}$ in

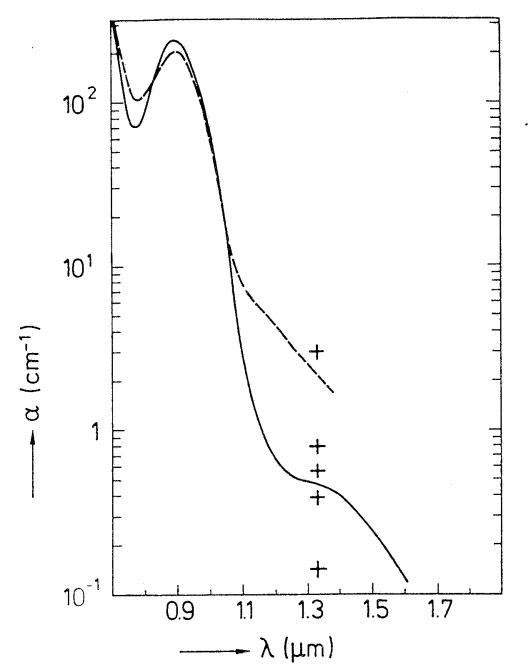

Fig. 7. Optical absorption spectra for flux grown YIG single crystal with $\mathrm{Pb}=0.012$ atoms/f. $u$. (solid curve), $\mathrm{Gd}_{2} \mathrm{Bi}_{1} \mathrm{Fe}_{4.4} \mathrm{Al}_{0.4}$ $\mathrm{Ga}_{0.2} \mathrm{O}_{12}$ LPE film with $\mathrm{Pb}=0.035$ atoms/f.u. and $\mathrm{Pt}=0.031$ atoms/f.u. (dashed curve) and $(\mathrm{Gd}, \mathrm{Bi})_{3}(\mathrm{Fe}, \mathrm{Al}, \mathrm{Ga})_{5} \mathrm{O}_{12}$ sputtered epitaxial film (crosses) (from Ref. 18)). which the rare earth ion is fully replaced by $\mathrm{Bi}$ is a typical example. This film has not been prepared by the LPE method as described above. The epitaxial films of $\mathrm{Bi}_{3} \mathrm{Fe}_{5} \mathrm{O}_{12}$ have been recently grown using ion-beam sputtering ${ }^{19)}$ and the conventional $\mathrm{rf}$ diode sputtering, ${ }^{20)}$ and ECR sputtering. ${ }^{21)}$ Figure 8 shows lower limit of substrate temperature $T_{\mathrm{g}}$ required for in situ epitaxially growing the Bi-substituted iron garnet $\left(\mathrm{Y}_{3-x} \mathrm{Bi}_{x} \mathrm{Fe}_{5} \mathrm{O}_{12} ; \mathrm{BiIG}\right)$ layer by the rf sputtering. The value of $T_{\mathrm{g}}$ is as low as $390^{\circ} \mathrm{C}$ for BiIG. The epitaxial BiIG films exhibit a narrow X-ray diffraction linewidth as shown in Fig. 9, even though the lattice mismatch between the film and the substrate is fairly large $(0.9 \%)$. They also show specific Faraday rotation as prominently large as $8.6 \times 10^{4} \mathrm{deg} / \mathrm{cm}$ at $\lambda=633 \mathrm{~nm}$, which is 100 times larger than that of pure YIG $(x=0)$ film (Fig. 10).

The BiRIG films can be prepared by a chemical method, i.e. pyrolysis as well. ${ }^{22)-24)}$ As shown in Fig. 11 , the surface of a glass substrate is spin-coated with a nitric acid solution of the garnet-constituting metals. The coating is dried at $150^{\circ}-400^{\circ} \mathrm{C}$ for evaporating the solvent and then decomposing the nitrates. Repeating the spin-dry process, we obtain an

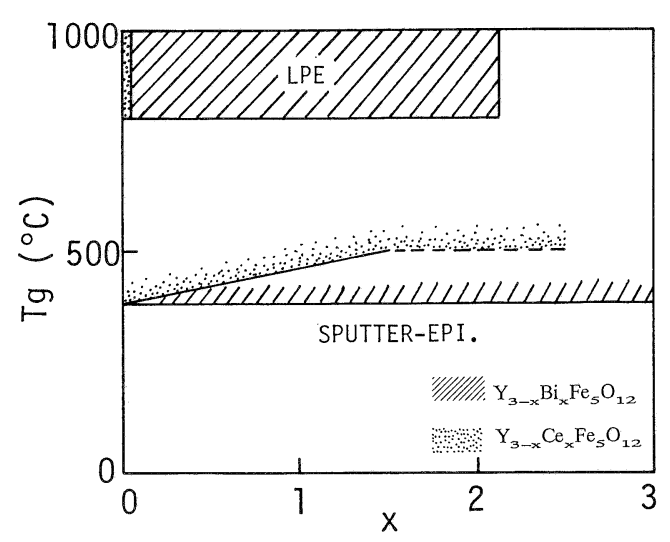

Fig. 8. Threshold substrate temperature $T_{\mathrm{g}}$ for in situ epitaxial growth of $\mathrm{Y}_{3-x} \mathrm{Bi}_{x} \mathrm{Fe}_{5} \mathrm{O}_{12}$ film and $\mathrm{Y}_{3-x} \mathrm{Ce}_{x} \mathrm{Fe}_{5} \mathrm{O}_{12}$ film by rf sputtering. Those by LPE method are shown for comparison.

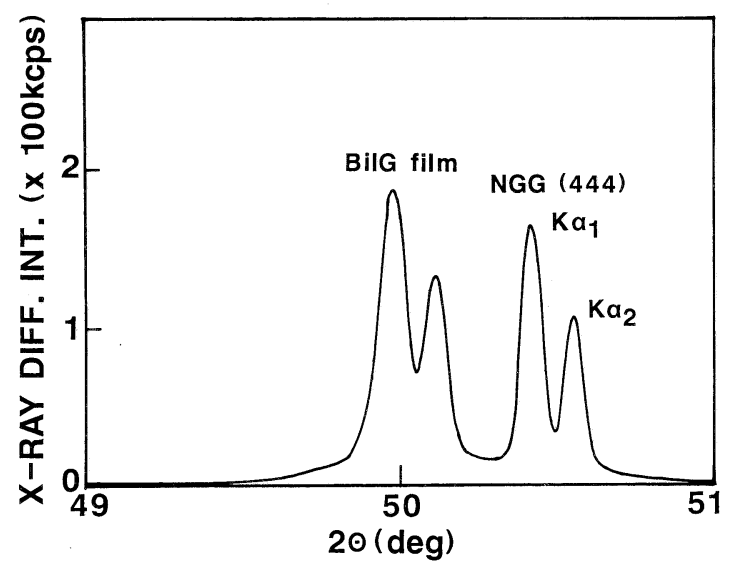

Fig. 9. X-ray diffraction diagram of sputtered epitaxial $\mathrm{Bi}_{3} \mathrm{Fe}_{5}$ $\mathrm{O}_{12}$ film in situ grown on (111) $\mathrm{NGG}$ at $T_{\mathrm{s}}=420^{\circ} \mathrm{C}$. 


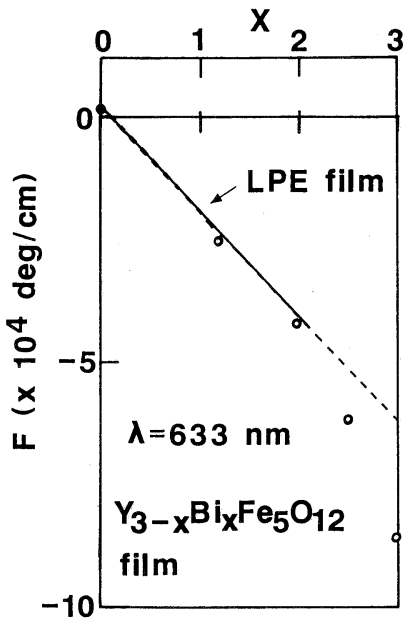

Fig. 10. Specific Faraday rotation $F$ of $\mathrm{Y}_{3-x} \mathrm{Bi}_{x} \mathrm{Fe}_{5} \mathrm{O}_{12}$ epitaxial films grown by sputtering (open circles) and by LPE (solid line). ${ }^{14)}$



Fig. 11. Schematic representation of the pyrolysis method.

amorphous oxide film of the desired thickness. The film is crystallized by heating in air at $550^{\circ}-700^{\circ} \mathrm{C}$. Using the same procedure we can grow the film on a single crystal wafer by solid phase epitaxy. This pyrolysis has following advantages over the sputtering: (1) The chemical composition in the film does not deviate from that in the target, because the ions in the starting solution do not leave the film appreciably, and (2) it does not require vacuum, thus favoring mass production. However, it should be noted that the film obtained by the pyrolysis may be somewhat porous because a part of decomposed gas products remains in the film.

\subsubsection{Light rare earth-substituted iron garnet films}

It is expected that light rare earth ions such as $\mathrm{Pr}^{3+}, \mathrm{Nd}^{3+}, \mathrm{Ce}^{3+}$ in garnet may prominently enhance the MO effect due to the $4 \mathrm{f}-5 \mathrm{~d}$ orbital promotion transitions located in the UV region, because the transitions have strong oscillator strength and large spin-orbit parameters at the excited states. Wemple et al. reported that garnet compositions cotaining $\mathrm{Pr}$ and $\mathrm{Nd}$ had large Faraday rotation. ${ }^{7)}$ However until recently it has only been possible to replace a fraction of the rare earth ion of the matrix with Pr and Nd. Recently Fratello et al. reported the first growth of PrIG film and NdIG film by the LPE at relatively low temperatures on substrates with large lattice parameters. ${ }^{25)}$

On the other hand, the rare earth iron garnet films containing a large amount of $\mathrm{Ce}^{3+}$ have not yet been grown by the LPE method. The achieved amount of Ce substitution is as small as less than 0.06 per a chemical formula unit. This is because ionic radius of $\mathrm{Ce}^{3+}$ is too large for the garnet lattice and $\mathrm{CeO}_{2}$ separates in the melted flux. ${ }^{26)}$ However, by rf sputtering, we recently succeeded in growing the epitaxial films of Ce-substituted iron garnet $\left(\mathrm{Y}_{3-x} \mathrm{Ce}_{x} \mathrm{Fe}_{5} \mathrm{O}_{12}\right)$ with so much substitution as up to $x=2.5$ at low temperature (Fig. 8) .27),28) This is also an example showing the advantage of the sputtering method superior to other methods.

The epitaxial film was found to strongly enhance the MO effect in the visible and near IR regions. ${ }^{27)}$ Figure 12(a) shows enhancement of Faraday rotation by $\mathrm{Ce}^{3+}$ substitution and that by $\mathrm{Pr}^{3+}$ substitution is shown in Fig. 12(b) for comparison. ${ }^{29)}$. The Pr substitution induces the pronounced Faraday rotation toward higher photon energy side, while Ce substitution prominently enhaneces Faraday rotation in the wide range of photon energy. The Ce contribution to Faraday effect shows paramagnetic dispersion centered at two energy $h v=1.4 \mathrm{eV}$ and $3.1 \mathrm{eV}$ as shown in Fig. 12.30) However, the mechanism of the enhancement is not clear at present: This enhancement occurs at too low photon energy range to be attributed to the preceding $4 \mathrm{f}-5 \mathrm{~d}$ electronic transition.

\subsubsection{Spinel ferrite and hexagonal ferrite films cotaining $\mathrm{Co}^{2+}$}

The developments of the films of spinel ferrite and hexagonal ferrite containing $\mathrm{Co}^{2+}$ have been for $\mathrm{MO}$ recording. ${ }^{31), 32)}$ This is because both films have strong optical absorptions and large coercive forces suitable for the MO recording. In MO recording medium the easy axis of the magnetization should be directed perpendicular to the film plane. In the polycrystalline ferrite films deposited on non-single crystalline substrate, we can get the perpendicular magnetic anisotropy by crystalline anisotropy and/or stress induced anisotropy. ${ }^{33)}$ The uniaxial magnetic anisotropy constant $K_{\mathrm{u}}$ due to the stress induced anisotropy is proportional to the magnetostriction coefficient $\lambda_{\mathrm{s}}$ of the polycrystalline film and the thermal stress $\sigma$ induced into the film, which is expressed as

$$
\begin{aligned}
K_{\mathrm{u}} & =-3 \lambda_{\mathrm{s}} \sigma / 2 \\
& =-3 \lambda_{\mathrm{s}}\left(\alpha_{\mathrm{f}}-\alpha_{\mathrm{s}}\right) E \Delta T / 2(1-v) .
\end{aligned}
$$




\section{$\lambda(\mathrm{nm})$}

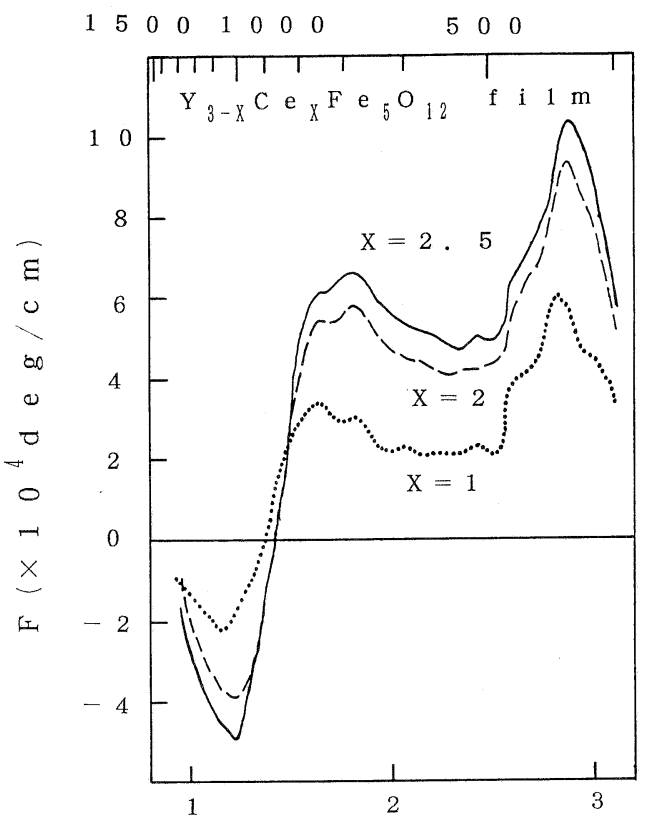

h $\nu($ e $V)$

(a)

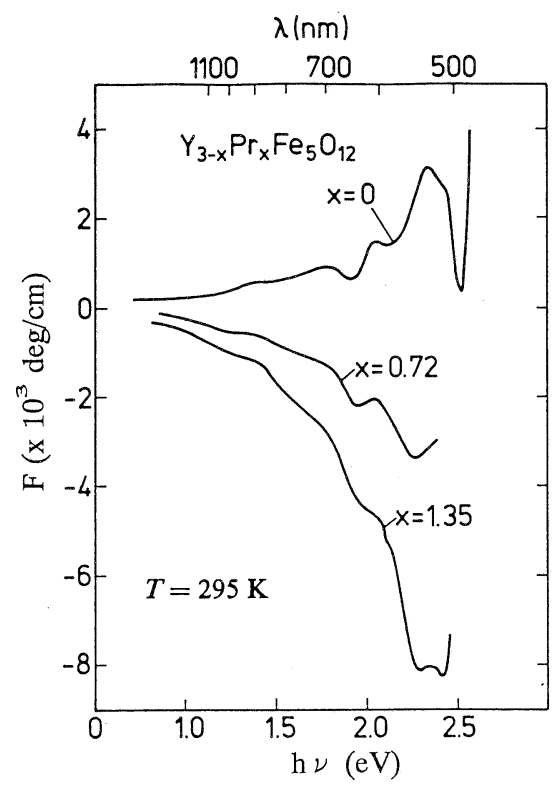

(b)

Fig. 12. Enhancement of Faraday rotation by (a) Ce substitution and (b) Pr substitution (from Ref. 29)).

Here, $\Delta T$ is difference between substrate temperature (at which the film is crystallized) and room temperature, $\alpha_{\mathrm{f}}$ and $\alpha_{\mathrm{s}}$ the thermal expansion coefficients of the film and the substrate, respectively, and $E$ and $v$ Young's modulus and Poisson's ratio, respectively, of the film.

We could obtain perpendicularly magnetized film for $\mathrm{CoCrFeO}_{4}$ spinel ferrite with $\lambda_{\mathrm{s}}>0$ on metal substrate $\left(\alpha_{\mathrm{f}}<\alpha_{\mathrm{s}}\right)$ using stress induced anisotropy. ${ }^{34)}$ Stronger perpendicular magnetic anisotropy can be obtained in the $\mathrm{CoFe}_{2} \mathrm{O}_{4}$ film with a preferred crystal orientation of $\langle 100\rangle$ axis normal to the film plane on a substrate, since the crytalline magnetic anisotropy constant $K_{\langle 100\rangle}$ in $\mathrm{CoFe}_{2} \mathrm{O}_{4}$ is positive and large, which, as well as the stress induced anisotropy, gives a positive contribution to the perpendicular magnetic anisotropy.

The $\mathrm{CoFe}_{2} \mathrm{O}_{4}$ films having a preferred crystal orientation of $\langle 100\rangle$ axis normal to the film plane have been successfully prepared on substrate by spray pyrolysis, ${ }^{31)}$ ion plating and the rf sputtering. The epitaxial films of $\mathrm{Co}_{x} \mathrm{Fe}_{3-x} \mathrm{O}_{4}^{35)}$ and $\mathrm{Co}_{x} \mathrm{Fe}_{3-x-y}$ $\mathrm{Cr}_{y} \mathrm{O}_{4}{ }^{36)}$ have been also grown on (100)-oriented $\mathrm{MgO}$ single crystal substrates by chemical transport reaction using a carrier gas of the mixture of dry $\mathrm{HCl}$ and $\mathrm{Cl}_{2}$. These films exhibited fairly large coercive forces associated with strong crystalline magnetic anisotropy, as well as large Faraday rotation as shown in Fig. 14.

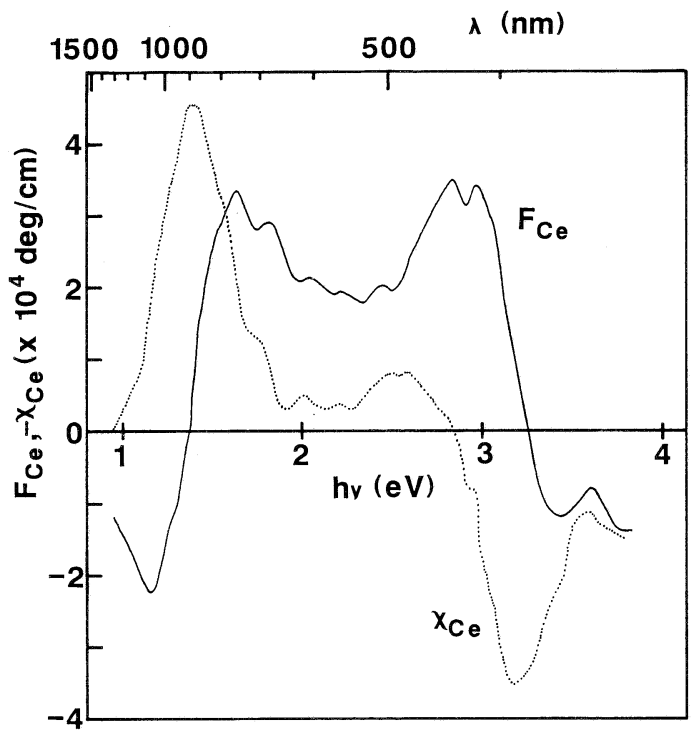

Fig. 13. Ce contribution to Faraday rotation $F_{\mathrm{Ce}}$ and Faraday ellipticity $\chi_{\text {Ce }}$.

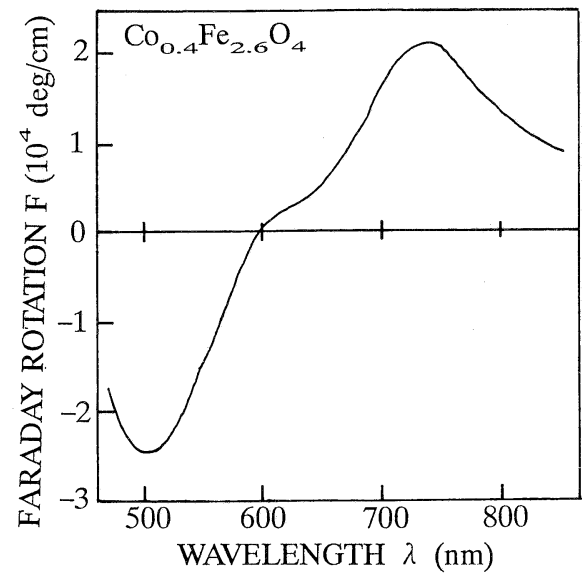

Fig. 14. Faraday rotation spectrum for $\mathrm{Co}_{0.4} \mathrm{Fe}_{2.6} \mathrm{O}_{4}$ film (from Ref. 36)). 


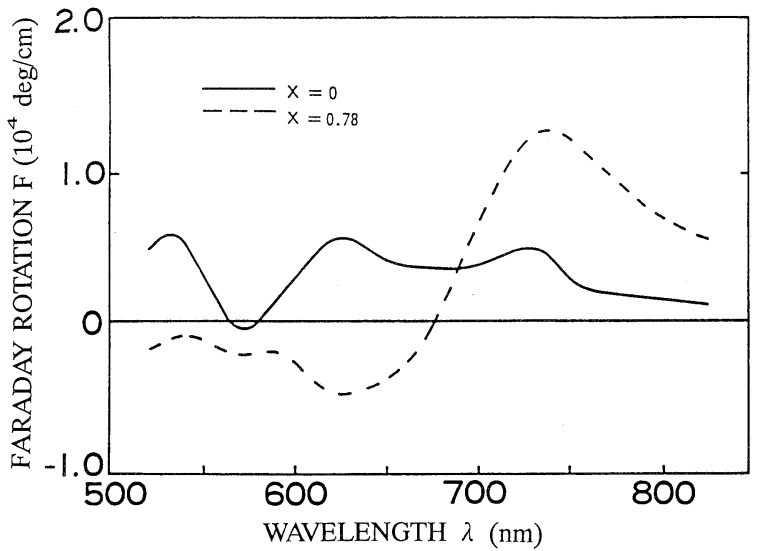

Fig. 15. Faraday rotation spectra for $\mathrm{BaFe}_{12-2 x} \mathrm{Co}_{x} \mathrm{Ti}_{x} \mathrm{O}_{19}$ films (from Ref. 32)).

Few works on hexagonal ferrite thin films for MO applications have been done as far as we know. The film of hexagonal ferrite $\mathrm{BaFe}_{12} \mathrm{O}_{19}$ and $\mathrm{Co}$, Ti-substituted $\mathrm{Ba}$ ferrite with a good orientation of $\langle 001\rangle$ axis normal to the film plane have been prepared on oxidized silicon wafers with $\mathrm{ZnO}$ layer on the top ${ }^{37)}$ and (111)-oriented single crystal GGG substrates ${ }^{32)}$ by targets-facing-type sputtering method. The easy axis of the magnetization is directed normal to the film plane by the large uniaxial magnetic anisotropy of the hexagonal ferrite. The Faraday rotation of the $\mathrm{Co}$, Ti-substituted hexagonal ferrite film increases in the IR region with Co content increasing as shown in Fig. 15. ${ }^{32)}$ This enhancement is caused by replacement of $\mathrm{Fe}^{3+}$ with $\mathrm{Co}^{2+}$ in spinel blocks of Ba ferrite unit cell, which is understood from the comparison with the Faraday spectrum of Co spinel ferrite (Fig. $14)$.

\subsubsection{Semiconductors}

A new class of ternary alloys called dilute magnetic semiconductors is known to show a large Faraday rotation at room temperature. These materials are basically II-VI semiconductors such as CdTe with a large fraction of the group II element substituted by a transition metal magnetic ion such as $\mathrm{Mn}$. The large Faraday rotation is ascribed to large Zeeman splitting in an external magnetic field which is enhanced by localized magnetic moment interacting strongly with band electrons by an exchange mechanism. The low optical absorption coefficient combined with a very large Faraday rotation makes these materials promising candidates for MO devices such as magnetic field sensor, isolator and modulators.

The single crystal films of $\mathrm{Cd}_{1-x} \mathrm{Mn}_{x} \mathrm{Te}$ with (111)-orientation were epitaxially grown on (100)oriented GaAs wafer and (0001)-oriented sapphire substrate by Molecular Beam Epitaxial method ${ }^{38)}$ and Ionized Cluster Beam method, ${ }^{39)}$ respectively. These films show so much small optical absorption coefficient at photon energy above band gap energy, therefore having a large MO figure of merit as shown in Fig. 16.

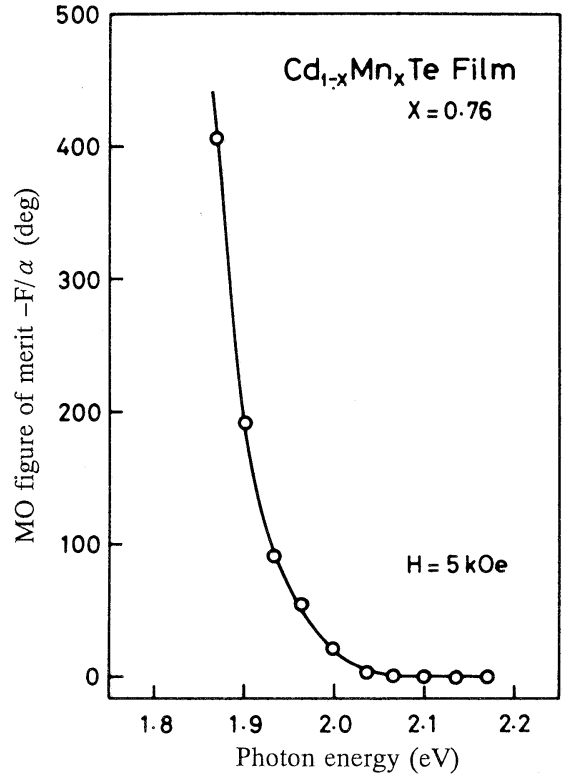

Fig. 16. Magneto-optical figure of merit for $\mathrm{Cd}_{1-x} \mathrm{Mn}_{x} \mathrm{Te}$ $(x=0.76)$ epitaxial film (from Ref. 39)).

\subsubsection{Metals}

Since only Kerr effect can be used in metal films exhibiting extremely strong optical absorption, the applications of MO metal thin films are limited to reflection-type optical devices such as MO recording medium. The following films have recently attracted much attention as the $\mathrm{MO}$ recording medium.

(a) Rare earth-transition metal (R-T) amorphous films

This film can be prepared at room temperature by vacuum evaporation method or sputtering method, which is characterized by large perpendicular magnetic anisotropy and high optical homogeneity, satisfying essentially a requisite for MO recording medium. However, it has also a significant drawback of low corrosion resistance. This problem is solved by sandwiching the film between some protecting layers. The Kerr rotation angle is as small as $0.3^{\circ}$ around the wavelength of $800 \mathrm{~nm}$ as shown in Fig. 17.40)

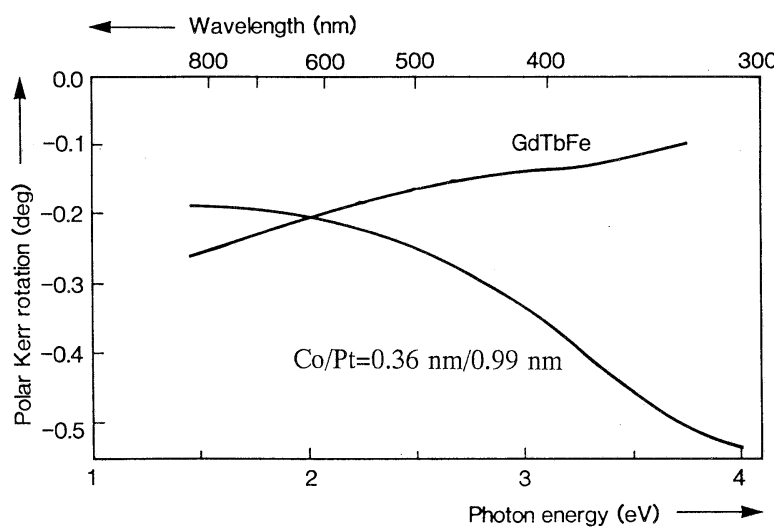

Fig. 17. Kerr rotation spectra for GdTbFe amorphous film and for $\mathrm{Co} / \mathrm{Pt}$ multilayer (from Ref. 40)). 


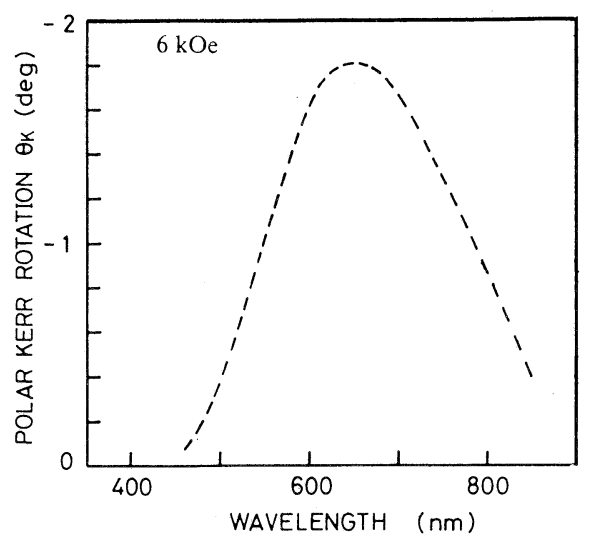

Fig. 18. Polar Kerr rotation spectrum in $6 \mathrm{kOe}$ for PtMnSb film annealed at $500^{\circ} \mathrm{C}$ (from Ref. 42 )).

(b) Co-Pt multilayers

This film was recently found to be a promising medium for high density MO recording using laser diode emitting short wavelength light such as blue light, which was prepared by alternately depositing $\mathrm{Co}$ and $\mathrm{Pt}$ using sputtering or evaporation with two sources. The film shows strong perpendicular magnetic anisotropy and high coercivity suitable for MO recording under a certain ratio of layer thickness between $\mathrm{Co}$ and $\mathrm{Pt} .{ }^{40)}$ The Kerr rotation angle increases at shorter wavelength region, larger than that of R-T amorphous films as shown in Fig. 17.

\section{(c) PtMnSb alloy films}

The film of PtMnSb which is a half metallic Heusler alloy with a $C_{1 \mathrm{~b}}$ structure has the highest MO Kerr effect of all known metallic systems at room temperature. ${ }^{41)}$ The Kerr rotation of the film is as large as $-1.8^{\circ}$ at $\lambda=633 \mathrm{~nm}$ as Fig. 18 shows. ${ }^{42)}$ These films are prepared on glass substrates at room temperature to $100^{\circ} \mathrm{C}$ by sequential depositions of each elements using electron beam evaporation ${ }^{42)}$ or by sputtering using complex targets. ${ }^{43)}$ They are followed by annealing above $500^{\circ} \mathrm{C}$ for crystallization.

\section{Summary}

We have outlined the phenomenological and microscopic theory of MO effect, which gives a criterion for researching new MO materials, and reviewed some recent works on the preparation and the MO properties of the magnetic thin films useful for MO applications. The substitution of specific ions such as $\mathrm{Bi}^{3+}$, light rare earth ions, and $\mathrm{Co}^{2+}$ to the ferrites strongly enhances MO effect, which is ascribed to the enhancement of spin-orbit interaction and/or oscillator strength in the electronic transitions. It has been emphasized that the preparation of the MO thin films by the sputtering method allows higher substitution of the specific MO enhancement ions than by other methods.

Acknowledgement The author would like to thank Prof. M. Abe for fruitful discussions and continuous encouragement.

\section{References}

1) R. L. Conger and J. L. Tomlinson, J. Appl. Phys., Suppl., 33, 1059 (1962).

2) M. Abe and M. Gomi, Oyobutsuri, 57, 723 (1988) [in Japanese].

3) J. C. Suits, IEEE Trans. Magn., MAG-8, 95 (1972).

4) J. L. Erskine and E. A. Stern, Phys. Rev. B, 8, 1239 (1973).

5) F. J. Kahn and P. S. Pershan, Phys. Rev., 186, 891 (1969).

6) H. Takeuchi, Jpn. J. Appl. Phys., 14, 1905 (1975).

7) S. H. Wemple, J. Electron. Mater., 3, 243 (1974).

8) R. Ahrenkiel and T. Coburn, IEEE Trans. Magn., MAG11, 1103 (1975).

9) K. Egashira, T. Manabe and H. Katsuraki, J. Appl. Phys., 42, 4334 (1971).

10) M. Abe and M. Gomi, J. Appl. Phys., 53, 8172 (1982).

11) M. Abe and M. Gomi, J. Magn. Magn. Mater., 84, 222 (1990).

12) H. Nelson, RCA Review, 24, 603 (1963).

13) R. C. Linares, R. B. McGraw and J. B. Schroeder, J. Appl. Phys., 36, 2884 (1965).

14) P. Hansen, K. Witter and W. Tolksdorf, Phys. Rev. B, 27, 6608 (1983).

15) E. Sawatzky and E. Kay, J. Appl. Phys., 39, 4700 (1968).

16) J.-P. Krumme, V. Doormann and R. Eckart, IEEE Trans. Magn., MAG-20, 983 (1984).

17) M. Gomi, T. Tanida and M. Abe, J. Appl. Phys.,57, 3888 (1985).

18) J.-P. Krumme, V. Doormann, B. Strocka and K. Witter, J. Appl. Phys., 62, 3879 (1987).

19) T. Okuda, N. Koshizuka, K. Hayashi, K. Satoh, H. Taniguchi and H. Yamamoto, J. Appl. Phys., 67, 4944 (1990).

20) M. Gomi, K. Satoh and M. Abe, Advances in Ferrites, Proc. 5th Int. Conf. on Ferrites, 919 (1989).

21) S. Mino, M. Matsuoka, A. Shibukawa and K. Ono, Jpn. J. Appl. Phys., 29, L1823 (1990).

22) M. Oron, I. Barlow and W. F. Traber, J. Mater. Sci., 4, 271 (1969).

23) T. Mizuno and M. Gomi, IEEE Trans. Magn., MAG-22, 1236 (1986)

24) J. Cho, M. Gomi and M. Abe, Jpn. J. Appl. Phys., 27, 2069 (1988).

25) V. J. Fratello, C. D. Brandle, S. E. G. Slusky, A. J. Valentino, M. P. Norelli and R. Wolfe, J. Cryst. Growth, 75, 281 (1986).

26) M. Kucera, J. Bok and K. Nitsch, Solid State Commun., 69, 1117 (1989).

27) M. Gomi, K. Satoh and M. Abe, Jpn. J. Appl. Phys., 27, L1536 (1988).

28) M. Gomi and M. Abe, Mat. Res. Soc. Symp. Proc., 150, 121 (1989).

29) P. Hansen, C.-P. Klages and K. Witter, J. Appl. Phys., 60, 721 (1986)

30) M. Gomi, H. Furuyama and M. Abe, Jpn. J. Appl. Phys., 29, L99 (1990).

31) J. W. D. Martens and A. B. Voermans, IEEE Trans. Magn., MAG-20, 1007 (1984).

32) H. Nakamura, F. Ohmi, Y. Kaneko, Y. Sawada, A. Watada and H. Machida, J. Appl. Phys., 61, 3346 (1987).

33) M. Abe and M. Gomi, Advances in Magneto-Optics, J. Magn. Soc. Jpn, 11, Suppl. No. S1, 299 (1987).

34) T. Yasuhara, M. Gomi and M. Abe, IEEE Trans. J. Magn. $J p n$, TJMJ-2, 750 (1987).

35） N. N. Evtihiev, N. A. Economov, A. R. Krebs, N. A. Zamjatina, V. D. Baurin and V. G. Pinko, IEEE Trans. Magn., MAG-12, 773 (1976).

36) I. S. Edelman and V. D. Baurin, Phys. Stat. Sol. (a) , 46, K83 (1978).

37) H. Machida, F. Ohmi, Y. Sawada, Y. Kaneko, A. Watada, and H. Nakamura, J. Magn. Magn. Mater., 54-57, 1339 (1986). 
38) L. A. Kolodziejski, T. Sakamoto, R. L. Gunshor and S. Datta, Appl. Phys. Lett., 44, 799 (1984).

39) T. Koyanagi and K. Matsubara, J. Appl. Phys., 61, 3020 (1987).

40) W. B. Zeper, F. J. A. M. Greidanus and P.F. Carcia, IEEE Trans. Magn., MAG-25, 3764 (1989).
41) P. G. Engen, K. H. J. Buschow and R. Jongebreur, Appl. Phys. Lett., 42, 202 (1983).

42) T. Inukai, M. Matsuoka and K. Ono, Appl. Phys. Lett., 49, 52 (1986).

43) R. Ohyama, T. Koyanagi and K. Matsubara, J. Appl. Phys., 61, 2347 (1987).

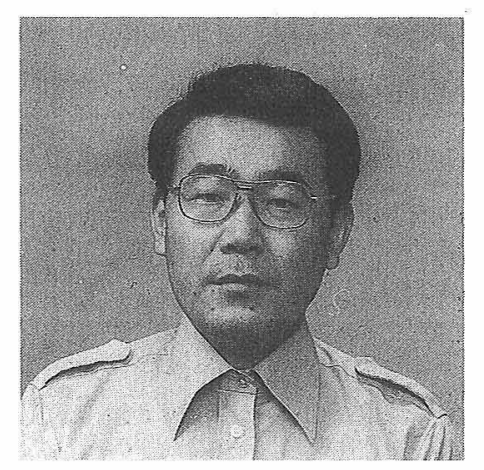

Manabu GOMI was born on Dec. 13, 1951 in Yamanashi Prefecture, Japan. He received the B. Eng. degree from the Yokohama National Univ. in 1974 and the Dr. Eng. degree from the Tokyo Institute of Technology in 1979. He is presently an associate professor of Department of Electrical and Electronic Engineering of Tokoy Institute of Technology. He is now working on synthesis and applications of new magneto-optical thin films. 